\title{
Dysregulation in Cortisol Diurnal Activity among Myeloproliferative Neoplasms Cancer Patients
}

\author{
Maricarmen Vizcaino ${ }^{*}$, Jennifer Huberty ${ }^{1}$, Linda Larkey ${ }^{2}$, Heidi Kosiorek ${ }^{3}$, Krisstina Gowin ${ }^{4}$, Ryan Eckert ${ }^{5}$ and Ruben A Mesa $^{5}$ \\ ${ }^{1}$ School of Nutrition and Health Promotion, Arizona State University, Arizona, USA \\ ${ }^{2}$ College of Nursing and Health Innovation, Arizona State University, Arizona, USA \\ ${ }^{3}$ Mayo Clinic, Phoenix, Arizona, USA \\ ${ }^{4}$ Department of Blood and Bone Marrow Transplant, University of Arizona, Tucson, USA \\ ${ }^{5}$ University of Texas Health San Antonio Cancer Center, USA
}

\begin{abstract}
Objective. Myeloproliferative neoplasms (MPNs) are rare hematological malignancies characterized by debilitating fatigue. Chronic stimulation of the hypothalamicpituitary-adrenal axis, due to cytokine overproduction and other factors during the cancer process, may represent one of numerous pathways leading to fatigue and decreased quality of life. The purpose of this study was to explore the characteristics of the diurnal cortisol response of MPN cancer patients and examine its association with self-reported physical and mental health.

Methods. Fifteen participants completed an online survey on physical and mental health, and collected saliva samples at home upon awakening, 30 minutes after waking, noon, and bedtime. Multiple indices of cortisol diurnal activity were estimated and compared against normative and flattened profiles published in a prior study on US adults, and their association with self-reported physical and mental health was explored.

Results. Area under the curve $\left(\mathrm{AUC}_{\mathrm{G}}\right)$ and cortisol awakening response (CAR) of MPN patients were significantly lower compared to normative values. Area under the curve $\left(\mathrm{AUC}_{\mathrm{G}}\right.$ and $\left.\mathrm{AUC}_{\mathrm{I}}\right)$, slope and evening cortisol were found to be significantly lower than flattened values. Additionally, a significant negative association was found between $\mathrm{AUC}_{\mathrm{G}}$ and perceived overall physical health.
\end{abstract}

Conclusion. A decline in total cortisol output may be indicative of physical health deterioration and a precursor of fatigue among MPN cancer patients; however, studies with larger samples are needed to confirm these results.

\section{Introduction}

Myeloproliferative neoplasms (MPNs), including polycythemia vera (PV), essential thrombocythemia (ET), and myelofibrosis (MF), are Philadelphia-negative clonal myeloid malignancies characterized by abnormal hematopoiesis, splenomegaly, risk of thrombotic and hemorrhagic sequelae, and risk of transformation to acute myeloid leukemia. Overall survival is significantly less compared to agematched controls $[1,2]$, but higher compared to many other cancers; patients can survive for several years and even a decade after diagnosis [3]. Survival years are characterized by severe symptom burden, from which the most common and debilitating is fatigue that contributes to diminished both physical and social functioning, and dramatically impacts quality of life [4].

Although the origin of fatigue may include multiple factors such as disease complications, comorbidities, psychological stress (e.g. anxiety, depression), and lifestyle habits [5], it has been suggested that fatigue may also have a pathophysiological origin. Cellular alterations that lead to cancer produce changes in cytokine expression, including abnormal cytokine production resulting from the body's inability to control cellular damage. Increased activity of tumor necrosis factor alpha (TNF- $\alpha$ ) has been associated with fatigue severity in women with breast cancer [6], whereas elevations in multiple cytokines including interleukin (IL)-6, IL-8, and TNF-alpha have been associated with symptomatology and disease progression across the Philadelphianegative MPN subtypes [7]. Nonetheless, the exact mechanism that explains the role of cytokines in the appearance and development of fatigue in cancer has not yet been fully elucidated.

Several cytokines, such as IL- 6 and TNF- $\alpha$, are known to stimulate the hypothalamic-pituitary-adrenal (HPA) axis [8] and when their activity is enhanced above normal conditions, such as in cancer, a chronic activation of the HPA axis may lead to exhaustion of adrenal glands or hypothalamic-medullar structures [9]. The subsequent HPA-axis burnout may result in underproduction of cortisol, which in turn has been associated with chronic and long-lasting fatigue in other medical conditions [10]. Furthermore, cortisol's main function is the restoration of homeostasis following stress exposure through a variety of actions including the inhibition of cytokine synthesis [11]; consequently, its limited availability may create a vicious cycle by contributing to additional cytokine production (Figure 1).

The HPA axis may be further overstimulated by sleep and psychological disturbances [12] known to be highly prevalent

${ }^{\star}$ Correspondence to: Maricarmen Vizcaino, School of Nutrition and Health Promotion, Arizona State University, 550 North 3rd St. Phoenix, AZ 85004, Arizona, USA, E-mail: mvizcain@asu.edu

Key words: cancer, cortisol, hypothalamic-pituitary-adrenal (HPA) axis, myeloproliferative neoplasms

Received: September 28, 2018; Accepted: October 09, 2018; Published: October 11,2018 


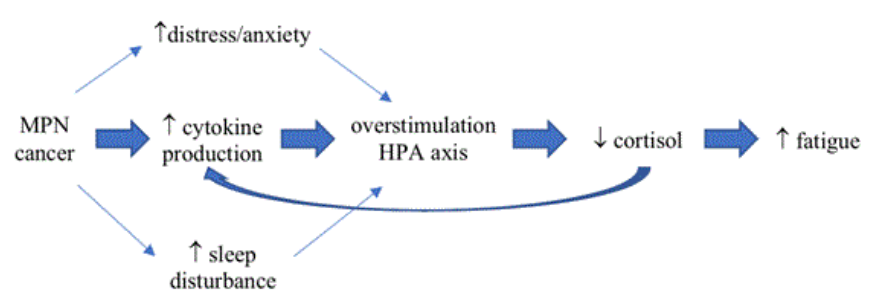

Figure 1. Hypothesized pathway linking cytokine production to fatigue in MPN cancer aggravated by sleep and psychological disturbances

among the Philadelphia-negative MPN subtypes [13,14], potentially contributing this way to the endless cycle of fatigue. The interaction between psychological and behavioral factors on neuroendocrine imbalances as part of a continuous cycle that is difficult to disrupt has been described before among breast cancer survivors [15].

An "exhausted" HPA axis, and subsequently an inability to adequately regulate the physiological response to stress, may represent one of various pathways contributing to the development of fatigue among MPN cancer patients. The temporal implication (i.e. underproduction of cortisol preceding fatigue) is supported by previous longitudinal research that found low waking cortisol levels and a flat diurnal cortisol slope to be significantly associated with new-onset fatigue 2-4 years later among healthy adults controlling for potential confounders (e.g. demographics, collection factors, behavioral factors, and measures of health) [16]. Nonetheless, this pathway has not been studied in MPN cancer patients.

Research exploring the interactions between cytokine and cortisol production in cancer-related fatigue is limited. However, the available evidence indicates that fatigued cancer patients do exhibit underproduction of cortisol during acute stress, which is in turn associated with elevated cytokines. Bower, et al. [17] found that breast cancer survivors with persistent fatigue did not exhibit the expected cortisol increase after The Trier Social Stress Test despite the presence of significant elevations in autonomic responses such as blood pressure and heart rate compared to non-fatigued cancer survivors showing a 4 -fold cortisol increase after the stress challenge.

Moreover, Bower, et al. [18] found a significant inverse association between cortisol production and IL-6 levels; that is, as cortisol increased, IL-6 levels decreased and as cortisol failed to increase, IL-6 levels increased. Further analysis of the data revealed that cortisol production, and not hormone sensitivity, predicted changes in IL-6, and hence they concluded that inadequate cortisol production may lead to exaggerated inflammatory responses and possibly contribute to a chronic inflammatory state in this subgroup of cancer patients [18].

An "exhausted" HPA axis may be further indicated by disruptions in cortisol diurnal activity. Normal cortisol secretion follows a daily pattern, with an abrupt increase 30 minutes following awakening and a subsequent decrease that continues throughout the day, reaching the lowest point at night $[19,20]$. Although a flattened profile has been observed in approximately $7-10 \%$ of adults, which is characterized by a small increase following awakening and elevated cortisol values throughout the day [20,21].

Multiple chronic conditions (e.g. fibromyalgia, chronic fatigue syndrome, diabetes mellitus) show disrupted patterns in cortisol diurnal activity, such as a combination of a flat slope and lower cortisol values, compared to what has been observed among the average adult population [22-24]. Similarly, dysregulated cortisol responses have been observed among ovarian and breast cancer patients including erratic peaks, a plateau with high values, and flatter slopes as compared to healthy controls $[25,26]$. Among cancer patients, those showing dysregulated cortisol diurnal activity report poorer self-perceived health and higher levels of fatigue [27-29]. Moreover, a flat diurnal cortisol rhythm has been identified as a significant and independent predictor of earlier mortality among lung and breast cancer patients after controlling for various markers of disease status, medical treatments, and psychosocial variables $[30,31]$.

It may be possible that alterations in cortisol diurnal activity are also present among other cancer populations, including those diagnosed with the Philadelphia-negative MPN subtypes; however, current literature has not described cortisol responses in this subgroup of cancer patients. Identifying dysregulations in cortisol diurnal activity may provide important insight into the health status of MPN cancer patients and allow an examination of the complex interplay between pathophysiological and psychological factors that affect circadian regulation and potentially contribute to the development of fatigue in MPN cancer. Thus, the purpose of this study was to explore the characteristics of the diurnal cortisol response among MPN cancer patients and its association with self-reported physical and mental health.

\section{Methods}

Data were derived from a previously published pilot study that examined the feasibility of remotely collecting biological samples prior to an online yoga intervention [32]. The study was approved by the institutional review board at a University in the Southwest United States. Patients previously diagnosed with the Philadelphia-negative classic MPNs (PV, ET, and MF) were recruited nationally through internetbased strategies (e.g. social media, email listervs) for participation in an online yoga intervention. Potential participants completed a brief questionnaire to determine eligibility for participation. Those who were eligible for the intervention and who were randomized to the online yoga group received an additional questionnaire to determine eligibility specifically for saliva cortisol collection.

In order to minimize the impact of behaviors/factors known to influence the cortisol response [33], a specific exclusion criteria was followed and included: 1) current use of steroid-base medications, 2) diagnosed endocrine disorders, 3) diagnosed mood disorders, 4) recent surgery that involved general anesthesia, and 5) current smoker. Only patients that were eligible for saliva cortisol collection were mailed a package that contained detailed instructions describing how to properly collect saliva at home in addition to all saliva collection materials.

Participants completed an online questionnaire via Qualtrics (Provo, UT) that assessed demographics (i.e., age, weight, height, income status, marital status, etc.), MPN-related health history (i.e., diagnosis, treatment history, current treatments, presence of anemia, etc.), and physical/mental health. Perceived overall physical health was assessed with the question "Considering your age, how would you describe your overall physical health?" and was answered in a scale from $1=$ Excellent to 5 = Poor). Self-rated health has been consistently found to be an independent predictor of mortality even after controlling for health practices and sociodemographic variables [34,35].

Anxiety, depression, sleep disturbance, and pain intensity were measured using the National Institutes of Health Patient Reported Outcomes Measurement Information System (NIH PROMIS) [36]. Each outcome was measured with items answered in a 5-point Likertscale. Items were summed across and the raw scores were converted to standardized t-scores using the publicly available NIH PROMIS scoring tools. A higher PROMIS score represents more of the concept 
being measured. The NIH PROMIS is a reliable and valid measure to assess patient-reported outcomes in cancer patients [37].

Fatigue was measured with the first question of the Myeloproliferative Neoplasm Symptom Assessment Form (MPNSAF) [38]. The question asks the respondent to rate fatigue experienced during the last 24 hours in a 10 -item scale ranging from $0=$ no fatigue to $10=$ worst imaginable. The MPN-SAF has been found to be valid and reliable in evaluating symptoms related specifically to MPN in multiple languages and with numerous populations.

Saliva collection. Participants were instructed to collect saliva at four different time points (upon awakening, 30 minutes postwaking, noon, and bedtime) over the course of one day prior to the yoga intervention. Before providing each sample participants were told to abstain from major meals, to avoid certain foods and liquids, to not brush teeth and to not rinse mouth. In a worksheet provided, participants reported the exact time of saliva collection for each time point in standard time, in addition to any oral health problems or acute illnesses. Participants used an oral swab (Salimetrics, LLC) for saliva collection and followed the instructions provided by the manufacturer, then stored collected samples in their personal freezer until shipment to the research staff. The times of saliva collection recorded on their respective worksheets indicated that instructions were followed within an acceptable margin. After receipt, samples were frozen and stored at $-80{ }^{\circ} \mathrm{C}$ at the ASU laboratory until shipment to the Institute for Interdisciplinary Salivary Bioscience Research (IISBR) at the University of California-Irvine (UCI) for cortisol content analysis. Collection of saliva samples at home has become standard protocol in cortisol research given the limitations of laboratory settings and the relevance of naturalistic conditions $[39,40]$ and is the same methodology followed by previously cited studies [25,29,31,41].

Cortisol analysis. On the day of the analysis, saliva samples were thawed completely and centrifuged at $1500 \mathrm{x}$ g for 15 minutes to precipitate mucins. Clear samples were added to a standard 96well plate and analyzed in duplicate using a highly sensitive enzyme immunoassay (Salimetrics, LLC). The test uses $25 \mu \mathrm{L}$ of saliva per determination, has a lower limit of sensitivity of $0.007 \mu \mathrm{g} / \mathrm{dL}$, standard curve range from $0.012 \mu \mathrm{g} / \mathrm{dL}$ to $3.0 \mu \mathrm{g} / \mathrm{dL}$, an average intra-assay coefficient of variation of less than 10 percent and an average interassay coefficient of variation less than 15 percent. Cortisol content was derived from the mean of the two results and reported in $\mu \mathrm{g} / \mathrm{dL}$.

Cytokine analysis. Blood samples were collected by Quest Diagnostics patient service centers (PSC) around the country. Participants were instructed to go to the nearest Quest Diagnostics PSC and receive a blood draw from a certified phlebotomist on staff. The obtained blood samples were analyzed for serum cytokines (IL-6, TNF- $\alpha$ ) content; results are reported in $\mathrm{pg} / \mathrm{mL}$.

Statistical analysis. Multiple indices of cortisol diurnal activity were estimated from the salivary samples obtained from MPN participants. For morning and night cortisol the samples collected immediately upon awakening and at bedtime were used respectively. Cortisol awakening response (CAR) was calculated by subtracting the immediately upon awakening value from the $30 \mathrm{~min}$ post-waking value. Area under the curve with respect to the ground $\left(\mathrm{AUC}_{\mathrm{G}}\right)$ and area under the curve with respect to increase $\left(\mathrm{AUC}_{\mathrm{I}}\right)$ were calculated using the trapezoidal formulas as suggested by the literature [42]; these methods provide an estimation of the total cortisol output and cortisol change over time, respectively. Cortisol slope was calculated by linearly regressing log transformed cortisol values to hours of the day when salivary cortisol was sampled.
Using the same calculations as above, normative indices were estimated from the normative cortisol profile published in a prior study [20]; values from each time point were converted from nmol/L to $\mu \mathrm{g} / \mathrm{dL}$, and then used to estimate cortisol indices. This normative profile was derived from a large sample of US adults between the ages of $34-87$ years, with $83 \%$ Caucasian, $10 \%$ Black, and $7 \%$ other racial origins; which is similar to the demographic characteristics of the MPN population [3]. In addition, because previous studies have observed that approximately $7-10 \%$ of adults show no discernible diurnal cycle, indices for the flattened profile also published by Dmitrieva, et al. [20] were calculated.

Data violated assumptions of normality as assessed by visual inspection of histograms and the Shapiro-Wilk test $(p<0.01)$, except for $\mathrm{AUC}_{\mathrm{G}}$. Log transformation failed to normalize the data, except for the slope. Hence, to compare indices of cortisol diurnal activity of MPN participants against the calculated normative and flattened indices, independent $\mathrm{t}$-tests were used for $\mathrm{AUC}_{\mathrm{G}}$ and slope, whereas onesample Wilcoxon signed rank tests were used for all the other cortisol variables. The association of cortisol diurnal activity with physical and mental health of MPN participants was probed through Spearman's rho correlational analyses between estimated cortisol indices and raw scores from cytokines (IL-6, TNF- $\alpha$ ), anxiety, depression, sleep disturbance, pain intensity, fatigue, and perceived physical health. Significance was set at alpha $<0.05$.

\section{Results}

Sixty-two MPN patients enrolled in the pilot study, 34 were randomized into the yoga group, 18 were eligible for saliva collection, and 15 returned saliva samples prior to the intervention. Demographic information on the patients that completed diurnal cortisol sampling is provided in Table 1. Five patients were diagnosed with PV, six with ET, and four with MF; the majority were diagnosed more than three years ago. All patients were White and non-Hispanic. The only common comorbidity among this group was high blood pressure, which was reported by $40 \%$ of patients $(n=6)$.

Physical and mental characteristics of MPN patients are presented in Table 2. Anxiety, depression, and sleep disturbance levels were within normal limits as compared to normative values in a general population. Pain intensity was slightly below the mean; the majority of patients described their pain as "mild" to "moderate" $(\mathrm{n}=12)$. Fatigue was slightly above the mean previously described in MPN populations; $M=5.7, S D=2.4$ vs. $M=4.4, S D=2.8$ [43]. None of the patients in this group had advanced to acute myeloid leukemia, none had their spleen removed, only five reported an enlarged spleen and only one reported severe bleeding in the past; however, several reported anemia $(n=9)$. The majority were taking medication including hydroxyurea $(\mathrm{n}=11)$, aspirin $(\mathrm{n}=12)$, interferon $(\mathrm{n}=3)$, anagelide $(\mathrm{n}=3)$, and ruxolitinib/ JAK2 inhibitor $(n=3)$. For additional MPN therapies used in the past, only one patient reported stem cell transplantation, one reported radiation, and half reported having a phlebotomy in the past $(n=7)$.

Visual inspection of individual cortisol curves (time of collection plotted against cortisol concentration) showed a flattened cortisol profile in five MPN patients that lack the typical rise observed 30 minutes after waking in healthy populations. The rest of the patients showed the same pattern of cortisol rising after waking and falling sharply afterwards with the lowest point at bedtime; however, values were consistently lower compared to the normative curve (Figure 2). Statistical analysis revealed that only the $\mathrm{AUC}_{\mathrm{G}}$ and CAR of MPN 
Table 1. Demographic characteristics of MPN patients completing saliva collection from online yoga intervention study

\begin{tabular}{|l|c|c|}
\hline \multicolumn{2}{|c|}{ Frequency } & Percent \\
\hline Age & 3 & 20.0 \\
\hline $40-50$ & 7 & 46.6 \\
\hline $51-60$ & 5 & 33.3 \\
\hline 61 and older & 3 & 20.0 \\
\hline Household income & 2 & 13.3 \\
\hline $21,000-40,000$ & 10 & 66.7 \\
\hline $41,000-60,000$ & & \\
\hline 61,000 and above & 2 & 13.3 \\
\hline Educational level & 2 & 13.3 \\
\hline High school & 3 & 20.0 \\
\hline Some college & 5 & 33.3 \\
\hline Associate's degree & 3 & 20.0 \\
\hline Bachelor's degree & & \\
\hline Graduate school or above & 13 & 86.7 \\
\hline Marital status & 2 & 13.3 \\
\hline Married & & \\
\hline Widowed & & \\
\hline
\end{tabular}

Table 2. Physical and mental characteristics of MPN patients completing saliva collection from online yoga intervention study

\begin{tabular}{|l|c|c|}
\hline & Mean & SD \\
\hline Overall physical health & 3.20 & 0.56 \\
\hline Anxiety & 50.79 & 8.26 \\
\hline Depression & 49.03 & 7.97 \\
\hline Sleep disturbance & 50.50 & 6.14 \\
\hline Pain intensity & 43.99 & 9.73 \\
\hline Fatigue & 5.73 & 2.43 \\
\hline
\end{tabular}

Note. Overall physical health was reported in a scale from $1=$ Excellent to $5=$ Poor. Anxiety, depression, sleep disturbance, and pain intensity were assessed with the NIH PROMIS questionnaire and are presented as t-scores, in which 50 is the mean of the population. Fatigue was assessed with the MPN-SAF TSS questionnaire and is presented as a raw score.

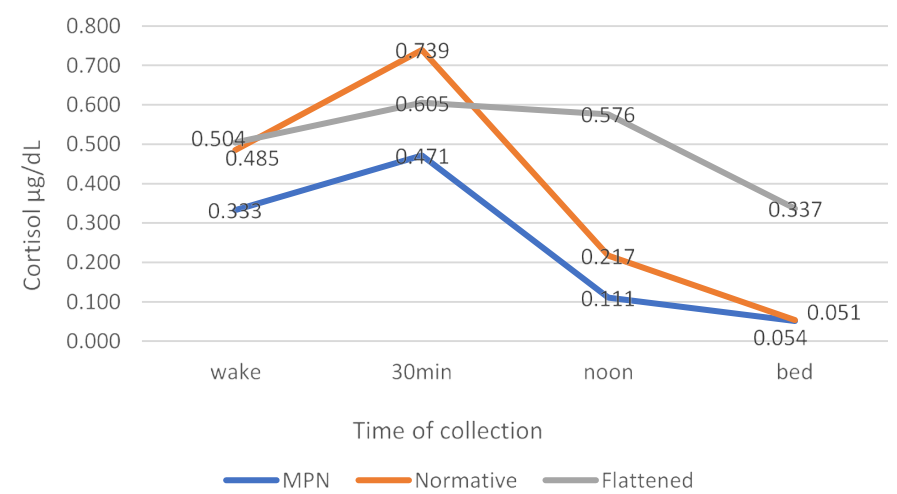

Figure 2. Cortisol diurnal rhythm of myeloproliferative neoplasms patients as compared to profiles derived from a US sample from the Dmitrieva, et al. study [20]

patients were significantly lower compared to the normative profile. On the other hand, $\mathrm{AUC}_{\mathrm{G}}, \mathrm{AUC}_{\mathrm{T}}$, slope, and evening cortisol of MPN patients were significantly lower compared to the flattened profile, whereas CAR and morning cortisol of MPN patients were not significantly different than flattened values (Table 3 ).

Spearman correlation analysis revealed a significant negative association between $\mathrm{AUC}_{\mathrm{G}}$ and perceived overall physical health $(r=$ $-0.53, p=0.04)$, indicating that those patients showing a lower cortisol output during the sampled day also reported the worst perceived overall physical health; recalling that participants described their physical health with a scale ranging from 1 (Excellent) to 5 (Poor). No significant associations were found between $\mathrm{AUC}_{\mathrm{G}}$ and the rest of the variables under investigation (all $p>0.09$ ) (Table 4 ).

The rest of cortisol indices (morning cortisol, evening cortisol, CAR, AUC $_{\mathrm{I}}$, cortisol slope) did not show significant associations with perceived physical health or any of the patient-reported physical symptoms. Anxiety was significantly associated with perceived physical health and depression; $r=0.55, p=0.03$ and $r=0.85, p<0.001$, respectively. Lastly, morning cortisol was significantly and negatively associated with $\mathrm{AUC}_{\mathrm{i}}(r=-0.97, p<0.00)$ and cortisol slope $(r=$ $-0.68, p<0.00$ ), while $\mathrm{AUC}_{\mathrm{i}}$ was significantly and positively associated with cortisol slope and CAR $(r=0.62, p=0.01$ and $r=0.53, p=0.04$, respectively).

\section{Discussion}

The present pilot study found a reduced $\mathrm{AUC}_{\mathrm{G}}$ and CAR among MPN cancer patients as compared to normative values derived from data previously published on US adults. In addition, the cortisol profile of MPN cancer patients was found significantly different from the flattened profile present among $10-17 \%$ of the general population as evidenced by both indices of AUC, slope and evening cortisol. These results may indicate that observed variations in the cortisol diurnal activity of MPN patients are the result of the disease and its physical and psychological disturbances, and not just due to uncommon variations found among the general population.

Suboptimal cortisol output may be an indication of the biological and psychological burden of cancer disease. Cellular alterations that give rise to inflammation, in addition to the mental stress during cancer diagnosis and treatment, may continually activate the HPA axis leading to "exhaustion" of adrenal glands. Subsequent underproduction of cortisol places the patient at risk for further inflammation during episodes of stress creating a vicious cycle (Figure 1). As previously explained before, this may represent one of several pathways that contribute to the development and perpetuation of fatigue among MPN cancer patients.

Previous studies have found dysregulation in the cortisol diurnal activity of cancer patients, which in turn has been associated to disease progression and worse prognosis. For example, elevated night cortisol was associated to high tumor grade among ovarian cancer patients [29], whereas a flattened cortisol slope was associated with early mortality among metastatic breast and lung cancer patients [25,30,31]. Participants in the present sample did not report evidence of advanced disease (e.g. post PV/ET myelofibrosis, acute myeloid leukemia, spleen removed, severe bleeding). This may explain the lack of disruption in other indices of cortisol diurnal activity as compared to the normative profile (morning cortisol, evening cortisol, $\mathrm{AUC}_{\mathrm{i}}$, and slope).

In addition, participants reported average levels of anxiety, depression, sleep disturbance, and pain intensity, as well as moderate symptoms of fatigue. Disruption of the HPA axis has been linked to psychosocial stress and stress-related bodily disorders (e.g. fibromyalgia). Hence, moderate symptoms of psychological/physical disturbances among the patients in this sample may not represent additional overload to the HPA axis further explaining unperturbed diurnal rhythm (i.e. slope, $\mathrm{AUC}_{\mathrm{I}}$ ).

In the present study, a significant negative association was found between total cortisol output and perceived overall physical health, that is, as the $\mathrm{AUC}_{\mathrm{G}}$ became lower participants reported poorer physical health (1= Excellent, 5= Poor). Similarly, Ho, et al. [28] found 
Table 3. Cortisol diurnal activity from MPN patients as compared to normative and flattened values from a US population sample

\begin{tabular}{|c|c|c|c|c|}
\hline Cortisol index & & & Difference from normative profile & Difference from flattened profile \\
\hline Morning cortisol $^{\mathrm{a}}$ & 0.33 & $(0.16,0.45)$ & 0.152 & 0.171 \\
\hline Evening cortisol $^{\mathrm{a}}$ & 0.05 & $(0.03,0.07)$ & 0.003 & $-0.287^{* *}$ \\
\hline $\mathrm{CAR}^{\mathrm{a}}$ & 0.02 & $(-0.04,0.26)$ & $0.234^{*}$ & -0.081 \\
\hline $\mathrm{AUC}_{\mathrm{G}}{ }^{\mathrm{a}}$ & 2.43 & 0.93 & $-1.813^{* *}$ & $-5.563^{* *}$ \\
\hline $\mathrm{AUC}_{1}^{\mathrm{a}}$ & -2.21 & $(-4.69,-0.77)$ & 1.210 & $2.180^{* *}$ \\
\hline Slope $^{\mathrm{b}}$ & -0.13 & 0.06 & 0.007 & $-0.104^{* *}$ \\
\hline
\end{tabular}

Note: $\mathrm{CAR}=$ cortisol awakening response, $\mathrm{AUC}_{\mathrm{G}}=$ area under the curve with respect to the ground, $\mathrm{AUC}_{\mathrm{I}}=$ area under the curve with respect to increase. Normally distributed variables are presented as mean \pm standard deviation; non-normally distributed variables are presented as median and interquartile range $\left(25^{\text {th }}, 75^{\text {th }}\right)$.

${ }^{\mathrm{a}} \mu \mathrm{g} / \mathrm{dL} .{ }^{\mathrm{b}} \log$ of $\mu \mathrm{g} / \mathrm{dL} .{ }^{*} p<0.05 ; * * p<0.01$

Table 4. Spearman correlation analyses between cortisol indexes and psychological/physiological parameters of MPN patients

\begin{tabular}{|c|c|c|c|c|c|c|c|c|c|c|c|c|c|c|}
\hline & $\begin{array}{c}\text { Cortisol } \\
\text { am }\end{array}$ & $\begin{array}{c}\text { Cortisol } \\
\text { pm }\end{array}$ & CAR & $\mathbf{A} \mathbf{U} \mathbf{G}_{g}$ & $\mathbf{A U C}_{\mathrm{i}}$ & Slope & $\begin{array}{c}\text { Physical } \\
\text { health }\end{array}$ & Anxiety & Depression & Sleep & fatigue & Pain & IL-6 & TNF- $\alpha$ \\
\hline Cortisol am & 1 & -0.202 & -0.415 & 0.450 & $-0.974 \dagger$ & $-0.685 \uparrow$ & -0.052 & 0.097 & 0.052 & 0.074 & -0.249 & -0.309 & 0.105 & -0.165 \\
\hline Cortisol pm & & 1 & -0.107 & -0.057 & 0.129 & $0.721 \uparrow$ & -0.441 & 0.004 & -0.220 & -0.155 & -0.079 & -0.245 & -0.028 & -0.252 \\
\hline CAR & & & 1 & 0.129 & $0.536^{*}$ & 0.311 & -0.116 & -0.339 & -0.393 & -0.450 & -0.226 & 0.140 & -0.392 & -0.172 \\
\hline $\mathbf{A U G}_{\mathrm{g}}$ & & & & 1 & -3.350 & -0.104 & $-0.530^{*}$ & -0.213 & -0.094 & 0.230 & -0.384 & -0.170 & -0.350 & 0.028 \\
\hline $\mathrm{AUC}_{\mathrm{i}}$ & & & & & 1 & $0.621^{*}$ & 0.000 & -0.175 & -0.128 & -0.138 & 0.210 & 0.248 & -0.238 & 0.214 \\
\hline Slope & & & & & & 1 & 0.019 & -0.130 & -0.105 & -0.131 & 0.127 & 0.288 & -0.133 & 0.270 \\
\hline Physical health & & & & & & & 1 & $0.555^{*}$ & 0.376 & 0.017 & 0.260 & 0.285 & 0.367 & 0.162 \\
\hline Anxiety & & & & & & & & 1 & $0.851 \uparrow$ & 0.222 & 0.354 & 0.403 & 0.481 & -0.294 \\
\hline Depression & & & & & & & & & 1 & 0.404 & 0.248 & 0.511 & 0.527 & -0.352 \\
\hline Sleep & & & & & & & & & & 1 & 0.435 & 0.079 & 0.113 & -0.166 \\
\hline fatigue & & & & & & & & & & & 1 & 0.241 & 0.050 & -0.145 \\
\hline Pain & & & & & & & & & & & & 1 & 0.544 & 0.166 \\
\hline IL-6 & & & & & & & & & & & & & 1 & -0.344 \\
\hline TNF- $\alpha$ & & & & & & & & & & & & & & 1 \\
\hline
\end{tabular}

Note: $\mathrm{CAR}=$ cortisol awakening response, $\mathrm{AUC}_{\mathrm{G}}=$ area under the curve with respect to the ground, $\mathrm{AUC}_{\mathrm{I}}=$ area under the curve with respect to increase, $\mathrm{IL}-6=$ interleukin 6 ; $\mathrm{TNF}-\alpha=$ tumor necrosis is factor alpha. "Statistically significant at alpha level 0.05 . $\uparrow$ Statistically significant at alpha level 0.01 .

a significant association between cortisol slope and perceived health among breast cancer patients controlling for age, cancer duration, and awakening time. These observations may indicate that as the physical health of cancer patients begin to deteriorate dysregulation in the diurnal cortisol activity starts to emerge.

Previous literature suggests that hypocortisolism or a hyporeactive HPA axis may result from prolonged periods of stress characterized by an initial hyperactivity of the HPA axis and excessive glucocorticoid release [44]. Therefore, dysregulations in total cortisol output among MPN patients may possibly appear in the early stages of disease, starting with a higher than normal $\mathrm{AUC}_{\mathrm{G}}$ and progressing to a lower than normal $\mathrm{AUC}_{\mathrm{G}}$. On the other hand, disruptions in the diurnal cortisol rhythm (e.g. slope, $\mathrm{AUC}_{\mathrm{T}}$ ) likely appear when the cancer has advanced into more aggressive stages or when the cancer has persisted for several years (as may be the case in MPN), leading to the emergence of debilitating physical symptoms like chronic fatigue. However, the current evidence is still inconclusive and additional studies are needed to support the present findings.

Some of the mechanisms that may be involved in the observed decrease in total cortisol output or deficiency of cortisol may include alterations along the HPA axis (e.g. reduced adrenocortical secretion, enhanced negative feedback inhibition), increased cortisol clearance, and/or reduced sensitivity of target cells for cortisol signaling [44]. Moreover, one important determining factor for the development of cortisol deficiency is the stressor characteristics [45]. Psychosocial and behavioral factors may play an important role in the appearance of cortisol diurnal dysregulations among MPN patients as they perceive and manage their disease in different ways leading to variations in the intensity and controllability of the stressor (cancer diagnosis and treatment).
Psychological and behavioral factors have been proposed to interact with neuroendocrine responses and ultimately determine health outcomes among cancer patients [15] and recent evidence may support this theory. For instance, among breast cancer patients perceived stress has been found negatively associated with $\mathrm{AUC}_{\mathrm{G}}$, indicating that as stress increases the total cortisol output decreases; whereas a "fighting spirit" as measured by the Mental Adjustment to Cancer Scale, has been found positively associated with $\mathrm{AUC}_{\mathrm{G}}$, indicating that as this positive construct increases the total cortisol output increases as well (46). Therefore, it is of crucial importance that clinicians consider the incorporation of therapies that can potentially enhance the psychological and physical well-being of patients.

\section{Limitations}

This pilot study has several limitations, including a small sample size composed of relatively "healthy" MPN patients. Studies with larger and more representative samples of MPN cancer patients are needed to confirm these findings. In may be possible that the observed perturbations in cortisol diurnal activity are characteristic of our particular sample; nonetheless, greater disturbance in cortisol diurnal activity may be expected from a more typical MPN patient sample given the high prevalence of fatigue and other physical symptoms in this cancer population [4].

Another limitation was a single-day measurement of cortisol given the feasibility nature of the pilot study from which the data derived. However, several studies examining diurnal cortisol rhythm in cancer patients have found no significant differences between sampling days $[28,47,48]$ and hence we feel that our data may still be a valuable first step in the examination of cortisol diurnal activity of MPN cancer patients. 
Finally, although a hypothetical pathway that may explain the appearance of fatigue among MPN cancer patients was presented, the observational and correlational nature of the study limits any causal inferences. It may be possible that the directional effect is reversed and fatigue, accompanied by perceived poorer physical health, leads to alterations in rest-activity cycles that in turn disrupt circadian rhythmicity including cortisol activity. Future research is needed to continue investigating the complex interaction between physiological/ psychological disturbances derived from the MPN cancer process, diagnosis, and treatment on the circadian rhythmicity and fatigue among patients.

\section{Future research suggestions}

Future studies should incorporate MPN patients with varied degrees of physical symptoms and disease status to allow for the exploration of changes in cortisol diurnal activity during cancer progression and treatment. For example, an important line of inquiry may be whether patients that have advanced towards the more symptomatic and worse prognosis of post PV/ET myelofibrosis or acute myeloid leukemia show greater cortisol dysregulation compared to patients that have maintained original diagnosis. In addition, future investigations should explore whether cortisol dysregulation differs between the different MPNs and what the implications may be for the patient's health status and subsequent medical treatment.

Lastly, it is of great importance that future research consider the standardization of cortisol measurement and detailed reporting of what was measured and how. Some previous studies have described the estimation of AUC without specifying whether the calculations were based on the formula with respect to the ground or with respect to increase, and these formulas indicate different concepts as previously mentioned. Standardization of cortisol measurement in cancer research will allow comparison of findings between studies, and possibly clarify whether discrepancy in results are due to measurement differences or due to inherent differences between cancer types, disease progression, and/or prognosis.

\section{Acknowledgements}

Philanthropic MPN funds were used to support this work.

\section{References}

1. Anía BJ, Suman VJ, Sobell JL, Codd MB, Silverstein MN, et al. (1994) Trends in the incidence of polycythemia vera among Olmsted County, Minnesota residents, 19351989. Am J Hematol 47: 89-93. [Crossref]

2. Mesa RA, Silverstein MN, Jacobsen SJ, Wollan PC, Tefferi A (1999) Population-based incidence and survival figures in essential thrombocythemia and agnogenic myeloid metaplasia: an Olmsted County Study, 1976-1995. Am J Hematol 61: 10-15. [Crossref]

3. Price GL, Davis KL, Karve S, Pohl G, Walgren RA (2014) Survival patterns in United States (US) medicare enrollees with non-CML myeloproliferative neoplasms (MPN). PloS one 9: e90299.

4. Mesa RA, Niblack J, Wadleigh M, Verstovsek S, Camoriano J, et al. (2007) The burden of fatigue and quality of life in myeloproliferative disorders (MPDs): an international Internet-based survey of 1179 MPD patients. Cancer 109: 68-76. [Crossref]

5. Scherber RM, Kosiorek HE, Senyak Z, Dueck AC, Clark MM, et al. (2016) Comprehensively understanding fatigue in patients with myeloproliferative neoplasms. Cancer 122: 477-485.

6. Bower JE, Ganz PA, Irwin MR, Kwan L, Breen EC, et al. (2011) Inflammation and behavioral symptoms after breast cancer treatment: do fatigue, depression, and sleep disturbance share a common underlying mechanism? J Clin Oncol 29: 3517-3522.

7. Geyer HL, Dueck AC, Scherber RM, Mesa RA (2015) Impact of inflammation on myeloproliferative neoplasm symptom development. Mediators Inflamm.
8. Haddad JJ, Saadé NE, Safieh-Garabedian B (2002) Cytokines and neuro-immuneendocrine interactions: a role for the hypothalamic-pituitary-adrenal revolving axis. J Neuroimmunol 133: 1-19.

9. Hänsel A, Hong S, Cámara RJ, Von Kaenel R (2010) Inflammation as a psychophysiological biomarker in chronic psychosocial stress. Neuroscience \& Biobehavioral Reviews 35: 115-121.

10. Bouillon R1 (2006) Acute adrenal insufficiency. Endocrinol Metab Clin North Am 35 767-775. [Crossref]

11. Sapolsky RM, Romero LM, Munck AU (2000) How do glucocorticoids influence stress responses? Integrating permissive, suppressive, stimulatory, and preparative actions. Endocr Rev 21: 55-89.

12. Buckley TM, Schatzberg AF (2005) On the interactions of the hypothalamic-pituitaryadrenal (HPA) axis and sleep: normal HPA axis activity and circadian rhythm, exemplary sleep disorders. J Clin Endocrinol Metab 90: 3106-3114.

13. McFarland DC, Polizzi H, Mascarenhas J, Kremyanskaya M, Holland J, et al (2016) Psychological Symptoms Among Patients With BCR-ABL-Negative Myeloproliferative Neoplasms. J Natl Compr Canc Netw 14: 1563-1570.

14. Gowin KL, Langlais BT, Kosiorek HE, Dueck A, Millstine D, et al. (2017) Sleep and Psychiatric Disturbance in Chronic Philadelphia Negative Myeloproliferative Neoplasms. Blood 130: 4189.

15. Larkey LK, Vega-López S, Keller C, McClain D, Ainsworth B, et al. (2014) A biobehavioral model of weight loss associated with meditative movement practice among breast cancer survivors. Health Psychol Open 1: 2055102914565495.

16. Kumari M, Badrick E, Chandola T, Adam EK, Stafford M, et al. Cortisol secretion and fatigue: associations in a community based cohort. Psychoneuroendocrinology 34 : 1476-1485.

17. Bower JE, Ganz PA, Aziz N (2005) Altered cortisol response to psychologic stress in breast cancer survivors with persistent fatigue. Psychosom Med 67: 277-280.

18. Bower JE, Ganz PA, Aziz N, Olmstead R, Irwin MR, et al. (2007) Inflammatory responses to psychological stress in fatigued breast cancer survivors: Relationship to glucocorticoids. Brain Behav Immun 21: 251-258.

19. Touitou Y, Sulon J, Bogdan A, Reinberg A, Sodoyez J, et al. (1983) Adrenocortical hormones, ageing and mental condition: seasonal and circadian rhythms of plasma 18-hydroxy-11-deoxycorticosterone, total and free cortisol and urinary corticosteroids. Journal of Endocrinology 96: 53-64.

20. Dmitrieva NO, Almeida DM, Dmitrieva J, Loken E, Pieper CF (2013) A day-centered approach to modeling cortisol: diurnal cortisol profiles and their associations among US adults. Psychoneuroendocrinology 38: 2354-2365.

21. Stone AA, Schwartz JE, Smyth J, Kirschbaum C, Cohen S, et al. (2001) Individual differences in the diurnal cycle of salivary free cortisol: a replication of flattened cycles for some individuals. Psychoneuroendocrinology 26: 295-306.

22. Nater UM, Youngblood LS, Jones JF, Unger ER, Miller AH, et al. (2008) Alterations in diurnal salivary cortisol rhythm in a population-based sample of cases with chronic fatigue syndrome. Psychosom Med 70: 298-305.

23. Bruehl H, Wolf OT, Convit A (2009) A blunted cortisol awakening response and hippocampal atrophy in type 2 diabetes mellitus. Psychoneuroendocrinology 34: 815-821.

24. Gur A, Cevik R, Nas K, Colpan L, Sarac S (2004) Cortisol and hypothalamic-pituitarygonadal axis hormones in follicular-phase women with fibromyalgia and chronic fatigue syndrome and effect of depressive symptoms on these hormones. Arthritis Res Ther 6: R232.

25. Abercrombie HC, Giese-Davis J, Sephton S, Epel ES, Turner-Cobb JM, et al. (2004) Flattened cortisol rhythms in metastatic breast cancer patients. Psychoneuroendocrinology 29: 1082-1092.

26. Touitou Y, Bogdan A, Levi F, Benavides M, Auzeby A (1996) Disruption of the circadian patterns of serum cortisol in breast and ovarian cancer patients: relationships with tumour marker antigens. Br J Cancer 74: 1248-1252.

27. Cuneo MG, Schrepf A, Slavich GM, Thaker PH, Goodheart M, et al. (2017) Diurnal cortisol rhythms, fatigue and psychosocial factors in five-year survivors of ovarian cancer. Psychoneuroendocrinology 84: 139-142.

28. Ho RT, Fong TC, Chan CK, Chan CL (2013) The associations between diurnal cortisol patterns, self-perceived social support, and sleep behavior in Chinese breast cancer patients. Psychoneuroendocrinology 38: 2337-2342.

29. Schrepf A, Thaker PH, Goodheart MJ, Bender D, Slavich GM, et al. (2015) Diurnal cortisol and survival in epithelial ovarian cancer. Psychoneuroendocrinology 53: 256-267. 
30. Sephton SE, Lush E, Dedert EA, Floyd AR, Rebholz WN, et al. (2013) Diurnal cortisol rhythm as a predictor of lung cancer survival. Brain Behav Immun 30 Suppl: S163-170. [Crossref]

31. Sephton SE, Sapolsky RM, Kraemer HC, Spiegel D (2000) Diurnal cortisol rhythm as a predictor of breast cancer survival. J Natl Cancer Inst 92: 994-1000. [Crossref]

32. Eckert R, Huberty J, Dueck A, Kosiorek H, Larkey L, et al. (2017) A Pilot Study of Online Yoga to Improve Fatigue and Quality of Life in Myeloproliferative Neoplasm Patients. Blood 130: 3443.

33. Stalder T, Kirschbaum C, Kudielka BM, Adam EK, Pruessner JC, et al. (2016) Assessment of the cortisol awakening response: expert consensus guidelines. Psychoneuroendocrinology 63: 414-432.

34. Idler EL, Benyamini Y (1997) Self-rated health and mortality: a review of twentyseven community studies. J Health Soc Behav 38: 21-37. [Crossref]

35. DeSalvo KB, Bloser N, Reynolds K, He J, Muntner P (2006) Mortality prediction with a single general self-rated health question. J Gen Intern Med 21: 267-275.

36. Hays RD, Bjorner JB, Revicki DA, Spritzer KL, Cella D (2009) Development of physical and mental health summary scores from the patient-reported outcomes measurement information system (PROMIS) global items. Qual Life Res 18: 873-880.

37. Quach CW, Langer MM, Chen RC, Thissen D, Usinger DS, et al. (2016) Reliability and validity of PROMIS measures administered by telephone interview in a longitudinal localized prostate cancer study. Qual Life Res 25: 2811-2823.

38. Scherber R, Dueck AC, Johansson P, Barbui T, Barosi G, et al. (2011) The Myeloproliferative Neoplasm Symptom Assessment Form (MPN-SAF): international prospective validation and reliability trial in 402 patients. Blood 118: 401-408.

39. Hofman LF1 (2001) Human saliva as a diagnostic specimen. J Nutr 131: 1621S-5S. [Crossref]
40. Saxbe DE (2008) A field (researcher's) guide to cortisol: tracking HPA axis functioning in everyday life. Health Psychology Review 2: 163-90.

41. Bower JE, Ganz PA, Dickerson SS, Petersen L, Aziz N, et al. (2005) Diurnal cortisol rhythm and fatigue in breast cancer survivors. Psychoneuroendocrinology 30: $92-100$.

42. Pruessner JC, Kirschbaum C, Meinlschmid G, Hellhammer DH. Two formulas for computation of the area under the curve represent measures of total hormone concentration versus time-dependent change. Psychoneuroendocrinology 28: 916-931.

43. Emanuel RM, Dueck AC, Geyer HL, Kiladjian JJ, Slot S, et al. (2012) Myeloproliferative neoplasm (MPN) symptom assessment form total symptom score: prospective international assessment of an abbreviated symptom burden scoring system among patients with MPNs. J Clin Oncol 30: 4098-4103.

44. Fries E, Hesse J, Hellhammer J, Hellhammer DH (2005) A new view on hypocortisolism. Psychoneuroendocrinology 30: 1010-1016. [Crossref]

45. Heim C, Ehlert U, Hellhammer DH (2000) The potential role of hypocortisolism in the pathophysiology of stress-related bodily disorders. Psychoneuroendocrinology 25: 1-35. [Crossref]

46. García J, Marisol D, Landero Hernández R, González Ramírez MT, Jaime Bernal L (2016) Diurnal cortisol variation and its relationship with stress, optimism and coping strategies in women with breast cancer. Acta Colombiana de Psicología 19: 113-122.

47. Kim KS, Kim YC, Oh IJ, Kim SS, Choi JY, et al. (2012) Association of worse prognosis with an aberrant diurnal cortisol rhythm in patients with advanced lung cancer. Chronobiol Int 29: 1109-1120. [Crossref]

48. Vedhara K, Tuinstra J, Miles JN, Sanderman R, Ranchor AV (2006) Psychosocial factors associated with indices of cortisol production in women with breast cancer and controls. Psychoneuroendocrinology 31: 299-311.

Copyright: (2018 Vizcaino M. This is an open-access article distributed under the terms of the Creative Commons Attribution License, which permits unrestricted use, distribution, and reproduction in any medium, provided the original author and source are credited. 